\title{
Les scènes du vomissement dans les tombes des nobles à Thèbes:
}

- Dr. Eman Ahmed Abou Zaid

\section{Préface:}

Les Égyptiens préféraient toujours la modération dans toute les choses, mais c'était possible de trouver dans les scènes des repas collectifs (banquet), l'un (une) des invités mange beaucoup ou boit jusqu'a l'ivresse et commence à vomir.

La recherche actuelle est une étude descriptive et analytique pour comprendre la nature et la signification des scènes du vomissement dans les tombes des nobles du Nouvel Empire à Thèbes. Nous allons recenser les tombes sur lesquelles figurent toutes les scènes qui restent soit bien visibles, soit moyennement conservées mais bien descriptibles et nous les classeront selon leur chronologie en précisant l'emplacement des scènes, les éléments de composition des scènes, la description des scènes. En conclusion, on essayeront d'arriver a la connaissance du symbolisme dans ces scènes, et surtout, l'artiste égyptien ancien qui s'intéresse à représenter l'élite de la société dans la forme idéale qu'ils veulent trouver dans l'au - delà. Donc, pourquoi sont-ils représentés dans cette état du vomissement?.

\section{(1): La définition du vomissement :}

\section{(A): La définition scientific:}

Le vomissement est le rejet actif par la bouche d'une partie du contenu de l'estomac. C'est une action protectrice de l'organisme qui a pour but de protéger ce dernier contre l'ingestion de substances toxiques. Il se distingue de la régurgitation qui elle est passive et est une simple remontée du contenu gastrique vers l'arrière-gorge ou la cavité buccale.

\footnotetext{
- Maître de Conférence- Faculté d’Archéologie - Université du Sud de la Vallée-Qena-Égypte.
} 


\section{دراسات في آثار الوطن العربي بـ}

Le mécanisme du vomissement est essentiellement d'origine centrale : dans le tronc cérébral se trouve le centre du vomissement recevant des informations du cortex, de la pression des ventricules cérébraux, des viscères et d'une zone de chimiorécepteurs. Il envoie des efférences vers le tube digestif, plus particulièrement vers le duodénum ${ }^{1}$.

\section{(B): La définition linguistique:}

Les dictionnaires de la langue égyptienne ancienne (hiéroglyphique) contiennent un certain nombre de significations des vocabulaires indiquant les vomissements

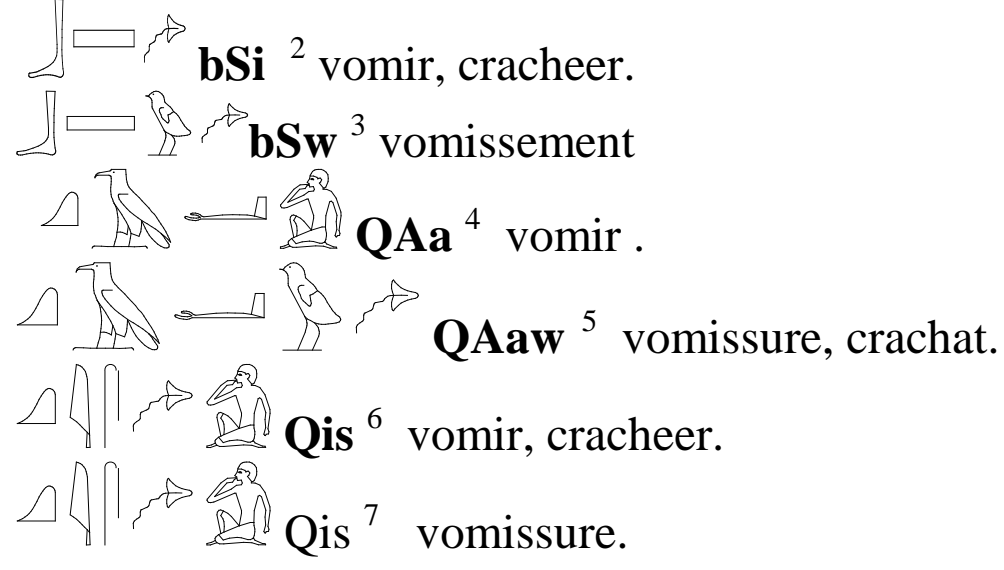

${ }^{1}$ Butte N., et al. ; Physiology of normal pregnancy; Le Caire, 2004; Borton C. \& Joshi A., Physiological changes in Pregnancy and Energy requirements during pregnancy based on total energy expenditure and energy deposition, le Caire, 2008.

${ }^{2}$ Wb I, 477 (17) il est apparu dans les texts des pyramides ; Meeks D., Année lexicographique, 2, Paris, 1981, (78.1371); Menu B., Petit Lexique de égyptienne hiéroglyphique, paris, 1989, p. 91.

3 Wb I, 478 (7). Il est apparu dans le Nouvel Empire (depuis la 18e - 20e dynastie) dans les texts magiques.

${ }^{4}$ Wb V, 7 (5) il est apparu dans le Moyen Empire, et parfois est écrit sans la lettre (a ) Qa, dans le copte dialecte Saydien il est écrit Kabol, et dans ló époque tardive il est écrit QAa, APA cf. Lesko L. H., A Dictionary of late Egyptian, U. S. A., 2004, P. 144.

${ }^{5} \mathrm{~Wb} \mathrm{~V}, 8(1)$, il est apparu dans les texts des pyramids et les papyrus médicaux; Meeks D., I, $o p$. cit., p. $383(77.4348)$.

${ }^{6} \mathrm{~Wb} \mathrm{~V}, 17$ (4-6), il est apparu dans les papyrus médicaux et lópoque Romaine, parfois il est écrit qAs

${ }^{7}$ Wb V, 17 (6), ce mots indique au nom du verbe vomir; Meeks D., 2, op. cit., p. 387 (78.4256). 
Il y avait aussi un autre mot

sri.t qui concerne le vomissement et il est apparu dans les papyrus médicaux Ebers ${ }^{8}$, Hearst ${ }^{9}$, Berlin ${ }^{10}$, mais comme une maladie, mais ce n'est pas notre sujet. Les méthodes de traitement trouvées dans ces papyrus médicaux étaient destinés pour soigner le vomissement comme une maladie ayant d'autres motifs (seryt) ${ }^{11}$, mais on n'y trouve aucun remède pour cet accidental vomissement: la raison principale était l'abondance alcoolique ${ }^{12}$ : expliqueé par les scènes étudiées. Une autre raison, c'est l'ajout du mandragore ${ }^{13}$ au vin qui mène au

${ }^{8}$ C'est le premier papyrus médical connu. Georg Ebers égyptologue allemand, se trouvait à Thèbes où il effectuait des fouilles durant l' hiver 1872-1873; il lui a acheté d'un égyptien qui déclara avoir découvert à Thèbes en 1862, entre les jambes d'une momie, et, quelque iours plus tard, Ebers pouvait déposer ce papyrus à l'université de Leipzig où il se trouve maintenant . Il est considéré le plus long document médical, il mesure 20,23 m de long et 0,30 de larg. Il donne un traité de physiologie et d'anatomie, cf. Leca A. P., Les medecine egyptienne au temps des pharaons, Paris, 1983, p. 21-30. Pour savoir plus cf. Ebbel B., The Papyrus Ebers, Londres, 1937; Chaliougui P., The Ebers Papyrus. A New English Translation, Commentaries and Glossaries, Le Caire, 1987.

${ }^{9}$ Découvert à Deir - el- Ballas en 1899, il est actuellement a l' université de Californie. Il daterait de la XVIII ${ }^{\mathrm{e}}$ dynastie du règne de Thoumosis III. «Il énonce de remèdes concernant l' intestin, le coeur, la vessie, la poitrine, les panaris et abcès dentaires, les panaris crocodil et de fauves. Il donne egalement des indications concernant 1 immobilisation des membres fractures». cf. Leca A. P., Ibid., p. 32. pour plus de details voir: Resner G.A., The Hearst Medical Papyrus ; Hieratic Text in 17 Facsimile Plates in Collotype with Introduction and Vocabulary, Egyptian Archeology, vol. I, Leipzig, 1905; Wreszinski W., Der Papyrus Hearst, Leipzig, 1902, p. 1-133.

10 «ll fut acquis à saqqara et publié en 1909 par Wreszinski, découvert dans une jarre enfouie a dix pieds sous tere, il est dans excellent etat de conservation. Il mesure $5,16 \mathrm{~m}$ de long sur $0,20 \mathrm{~m}$ de large, il daterait de la XIX ${ }^{\mathrm{e}}$ dynastie. Il contient des remedes contre les parasites intestinaux, les maladies des seins, la toux, les hematuries, les douleurs des membres inférieurs. Il est actuellement au musée de Berlin 3038». cf. Leca A. P., Ibid., p. 31; Wreszinski W., Der Grosse medizinische papyrus des Berline Museums, Leipzig, $1909 .$.

${ }^{11}$ Ebers (305-325), Hearst (62), Berlin (29-47) cf. Bardinet T., Les papyrus médicaux de l'égypte pharaonique, Paris, 1995, p. 298-300, p. 382, p. 413-415.

12 Brunner H., «Trunkenheit », LÄ VI, col. 773-777; Ikram S., «Banquets», in The Oxford Encyclopedia of Ancient Egypt 1, Oxford, 2001 , p 164; Wilfong T. G., «Intoxication » in Redford D. B. (éd.), The Oxford Encyclopedia of Ancient Egypt I, Oxford, 2001, p. 181; Erman A., Ranke H., La civilisation Egyptienne, (traduit par ) Mathien Ch., Paris, 1990, p.323

${ }^{13}$ La mandragore est une plante originaire du moyen orient (la Palestine, le Liban, la Syrie.), appartenant à la famille des solinacées. Elle a été introduite en Egypte vers le début du Nouvel Empire, par la reine Hatshepsout (cf., L. Keimer, " "La baie qui fait aimer", Mandragora officinarum L. dans l'Égypte ancienne », BIE32, 1951, p. 391.). Elle était mentionnée dans= 
vomissement ${ }^{14}$, peut-être, le vin contient aussi une matière sucrée ou plus concentrée: une mixture d'herbes ${ }^{15}$.

(2): Sources de l'Etude selon l'ordre chronologique:

(A): Les scènes du vomissement dans les tombes des nobles de la XVIII ${ }^{\mathrm{e}}$ dynastie:

(1): La Tombe d'Amenemhat ${ }^{16}$ Imn-m-HAt TT $53^{17}$ : DATE : Règne de Thoutmosis III. SITE : Cheikh Âbd el-Gournah.

BIBLIOGRAPHIE : Inpubliée sauf les photos de Chic.Or.Inst. (n ${ }^{\circ}$ 6421-23); Schott S., Däs Schöne Fest vom Wüstentale, Wiesbaden, 1952, tf. 11.

PLAN : $P M$ 1, p. 90.

=les textes sous le nom de rrmt surtout dans les chants d'amour. (Cf., Ph. Derchain, « Le lotus, la mandragore et le perséa », $C d E$ 50, p. 85. Le même mot est traduit dans Wb II, p. 439, comme : "Art Kleine Frucht... auch bei KränzenVerwendet, auch von der Brustwarze".

${ }^{14}$ Manniche L., "The so-called scenes of daily life in the private tombs of the Eighteenth Dynasty: an overview » in N. Strudwick, Taylor J. H. (éds.), The Theban Necropolis. Past, Présent and Future, British Museum Press, Londres, 2003, p. 44.

15 Manniche L., «Reflections on the Banquet Scene», in Tefnin R. (éd.), La peinture égyptienne ancienne. Un monde de signes à préserver, Actes du colloque international de Bruxelles, Avril 1994, Bruxelles, 1997, p. 33.

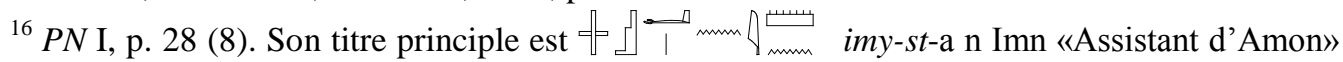
Urk IV, 1224 (9); W. A. Ward, Titles, p. 55, n434; A. Gardiner \&A. Weigall, Topographical Catalogue, p. 20; J. A. Taylor, Titles, p. 61 (558); S. Quirke, Titles and Bureaux of Egypt 18501700 BC, Londres, 2004, p. 127. Pour savoir la signification de ce titre de manière plus approfondie, et ses autres titres, cf., Awadalla A., «Un stele d'Amenemhat imy-st-a n Imn», BIFAO 89, 1989, p. 41, n. 13; Urk IV, 1217-25. ses parents sont 1 it nfr, 7 im $\boldsymbol{n} \boldsymbol{T r}$, sa femme est 


\section{دراسات في آثار الوطن العربي بر 1}

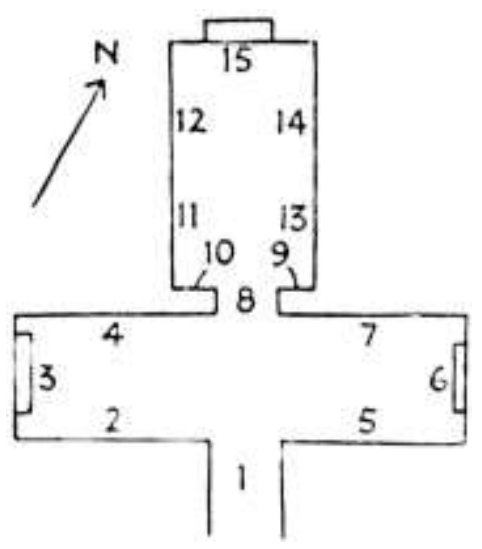

EMPLACEMENT DE LA SCÈNE: Sur la paroi Est, moitié Sud $\left(\mathrm{n}^{\circ} 2\right)$ de la salle transversale (pl. 1).

LA DESCRIPTION: Dans le troisième registre de la scène du banquet on voit un des invités assis sur un siège à haut dossier dont les pieds sont en forme de pattes de lion ${ }^{18}$ posé sur un socle ${ }^{19}$ posé ensuite sur une natte ${ }^{20}$. Il est torse nu, sauf un collier ousekh ${ }^{21}$

${ }^{18}$ Dans sa conclusion, C. De Wit (Le rôle et le sens du lion dans l'Égypte ancienne, Leiden, 1951) pense que le lion est un gardien. Il écarte le mal. Les pieds de sièges disposés en deux paires de pattes de lions sont l'image de l'animal protecteur du défunt. Au sujet de ce symbolisme voir. Pongracz M., «Löwendarstellungen an Podesten des Königsthrones», in: MDAIK 15, Le Caire, 1939, p. 213 f.; Schäfer H., " Altägyptische Bilder der auf- und untergehende Sonne», in: Z̈̈S 71, 1970, p. 15.

19 Les pieds des sièges de l'ancien Empire reposent sur de petits socles qui on des formes variées et sont exécutés dans des materiaux divers. Le rôle de socle était de cosolider et de protéger des coups la fragile extrémité sculptée du pied cf. Cherpion N., Mastabas et hypogées d'Ancien Empire. Le problème de la datation, Bruxelles, 1989. p. 36.

${ }^{20}$ Ils sont constituées de papyrus qui rappelle Osiris «car il sert à écrire le mot ouadj qui signifie vert, végétation et évoque le renouveau de la nature après l'inondation, de la même façon qu'Osiris a été rendue à la vie par l'action d'Isis »cf. Id., Deux tombes de la XVIIIe dynastie à Deir el Medina $N^{\circ} 340$ (Amenemhat) et 354 (anonyme), MIFAO 114,1999, p. 99. La natte sur laquelle sont assis les convives dans un banquet ou bien sur laquelle sont posés les sièges a pour rôle non seulement de les protéger de la poussière et des insectes mais surtout et en raison du papyrus qui la constitue d'offrir la renaissance. cf., Ibid., p. 100. La natte n'est pas réservée à tous les personnages représentés car dans les scènes du banquet, elle s'arrête sous les pieds de certains mais on ne la voit jamais sous les pieds des servantes et des serviteur. Appelé parfois collier ouah plutôt que ousekh. Il lié aux concepts de renaissance et de régénération. cf. Cherpion N, Ibid., p.100; selon Bruyère B. \& Kuentz. Ch., La tombe de Nakhtmin et la tombe d'Ari Nefer, MIFAO 54, Le Caire, 1926, p. 20 « le collier ousekh est un= 
autour de son cou. Il ne porte qu' un pagne court, une perruque courte et arrondie tombant sur la nuque découvrant les oreilles. Il tourne sa tête en arrière pour vomir.

On voit le vomissement sortant de sa bouche . Nous voyons aussi sa main droite a peine approche un pot posé derrière le siège, peut-être ce pot est déposé spécialement pour ce but, sa main gauche est tenue par son serviteur, débout devant lui pour l'aider (on voit une lacune entre les deux), tout en posant sa main droite sur l'épaule de son maître pour le supporter pendant le vomissement. Le buste du serviteur est aussi nu, sauf un collier ousekh autour de son cou, Il ne porte qu'un pagne court, une perruque courte et arrondie qui laisse degager les oreilles. Il est à noter que tous les deux ont les cônes d'onguent ${ }^{22}$ sur leurs têtes.

$$
\text { (pl. 1). }
$$

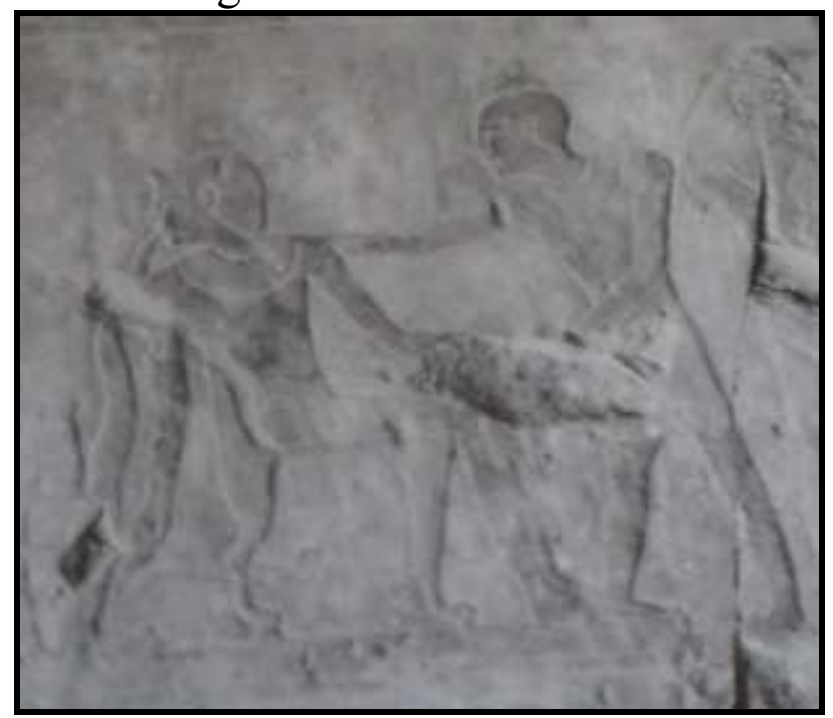

(2): La Tombe d' Iamounejeh

$i A m \cdot w-n D H{ }^{23} T T$ 84:

=des attributs d'Osiris que les mortels portent lorsqu' ils s'assimilent à lui par la justification d'outre tombe ou' sur cette terre, par la participation à une des ceremonies de son culte».

22 Cf. Ci-dessous, p. 11, n. 24.

${ }^{23}$ PN I, p. 6 (27). Ses titres principles sont $\neq$ की Whm tpy nsw «Premiere messager royal», imy $\mathrm{r}$ rwy $\mathrm{t}$ «Surveillant de la porte»cf. Gardiner A. \& Weigall A., op. cit., p. 20. = 


\section{دراسات في آثار الوطن العربي بـ}

DATE : Règne de Thoutmosis III.

SITE: Cheikh Âbd el-Gournah.

BIBLIOGRAPHIE: PM I, p. 167 (7) Inpubliée, sauf la photos de la chercheuse.

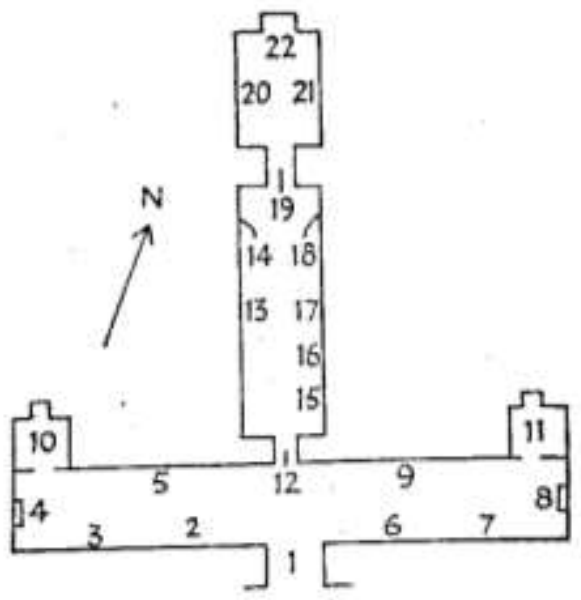

EMPLACEMENT DE LA SCÈNE: Sur le Mur Sud, moitié Ouest $\left(\mathrm{n}^{\circ} 7\right)$ de la salle transversale (Pl. 2).

LA DESCRIPTION : Dans le troisième registre de la scène du banquet on voit un des invités assis le deuxième. Il tourne sa tête en arrière pour vomir. On voit le vomissement sortant de sa bouche. Un serviteur (dans en mauvais état de conservation) se tient devant lui pour lui porter secours en lui posant une main sur la tête et l'autre main est effacée. L'invité qui assis derrière lui semble indifférent à ce qui se passe à côté de lui. Sa main droite ramenée à la poitrine tenant une grande fleur de lotus ${ }^{24}$ épanouie qu'il respire,

=au sujet des tous les titres cf. L. D. Text, III, p. 278 (13); Urk IV, 956-62 (278). Ses parents sont 1 i sA Dhwty ; rsy , sa femme est $\& 8 \square$ t

${ }^{24}$ le lotus était considéré comme source de vie permanente, et était devenu un symbole de renaissance La fleur de lotus par son parfum enivrant a un pouvoir magnétique se répandant à l'ensemble de ce qui l'entoure. En effet, et pour cette raison le défunt et les invités dans un banquet sont représentés dans les scènes en train de respirer l'odorant parfum, comme s'ils y puisaient le philtre d'une éternelle jouvence. Cf. Khattab H., «Le lotus bleu », in Parfums \& cosmétiques assoiffées et espoir aux gens dans l'Egypte ancienne, le Caire, Paris, Marseille,= 
alors que l'autre tient un linge plié. Devant lui, a été posée une table d'offrandes en albâtre, à pied central vert sur laquelle on voit une variété de nourriture est posée sur une natte (deux pains ronds et un ovale décorés sur les deux extrémités ${ }^{25}$, une grappe de raisins), tout est surmonté d'une botte d'oignons. Peut-être pour cette raison l'artiste présente le vomi se dirige vers 1 avant et n'est pas descedant par terre.

Celui qui vomis et l'autre invité sont assis sur des tabourets identique ${ }^{26}$, posé sur une natte. La partie supérieure du corps est nue. Sauf un collier ousekh ${ }^{27}$ autour de son cou. Il ne porte que deux pagnes superposés, un court à devanteau triangulaire et un long transparent ${ }^{28}$, autour de la taille, il y a une ceinture festonnée, une perruque courte et arrondi tombant sur la nuque découvrant les

=2002, p. 64 ; Ryhiner M.-L., l'offrande du lotus dans les temples égyptiens de l'époque tardive, Bruxelles, 1986, p. 222; Charron A., «L'image des cosmétiques chez les Egyptiens », in: Parfums \& cosmétiques dans l'Egypte ancienne, le Caire, Paris, Marseille, 2002, p. 68; Derchain Ph., «Le lotus, la mandragore et le perséa », CdE 50, p. 85.

${ }^{25}$ Le pain était et est encore de nos jours l'aliment essentiel au cours du repas et sa présence sur la table est obligatoire. Cependant, dans l'Egypte ancienne, il n'est pas uniquement un met, mais il est également utilisé dans d'autres circonstances telles que les rites religieux, les médecines, la magie. Le pain joue un grand rôle dans la vie économique par son utilisation quotidienne par son utilisation dans le troc et les échanges, il servait également de salaire pour les ouvriers, les soldats et les fonctionnaires .cf. Kemp B. J., Ancient Egypt. Anatomy of a Civilization, Londres, 1989, p. 126 ; D. Samuel, «Brewing and baking » in Nicholson P. T., \& Shaw I. (éds.), Ancient Egyptian Materials and Technology, Cambridge, 2000, p.. 537-576. Il en existe une multitude de variétés . Au sujet de tous les détails sur le pain cf. Almahdy E., Le Pain dans l'Égypte ancienne Jusqu'à la fin du Nouvel Empire, Thèse du DÉA, inpublié, (en arabe), Le Caire, 1995.

${ }^{26}$ le tabouret est de forme cubique de hauteur moyenne et fait de roseaux rassemblés entre eux ; cette forme rappelle le hiéroglyphe (P) cf. Davies N. de G., Gardiner A., The Tomb of Amenmenhet $\left(N^{\circ} 82\right)$, Londres, 1915, p. 64.

27 Appelé parfois collier ouah plutôt que ousekh. Il lié aux concepts de renaissance et de régénération. cf. Cherpion N, Ibid., p.100; selon B. Bruyère. Ch. Kuentz. La tombe de Nakhtmin et la tombe d'Ari Nefer, MIFAO 54, Le Caire, 1926.p. 20 "le collier ousekh est un des attributs d'Osiris que les mortels portent lorsqu' ils s'assimilent à lui par la justification d'outre tombe ou ' sur cette terre, par la participation à une des ceremonies de son culte»

${ }^{28}$ l'artiste utilise du rose pâle par opposition aux zones d'un blanc opaque qui indiquent une double epaisseur de tissu. 
oreilles, sur la quelle est posé un cône d'onguent ${ }^{29}$ en forme d'un demi cercle très peu élevé ${ }^{30}$. On observe que cet homme met ses deux mains (ne reste que des pripes de la main gauche et l'autre main est endommagée) sur son stomac peut être à cause de la forte du vomissement.

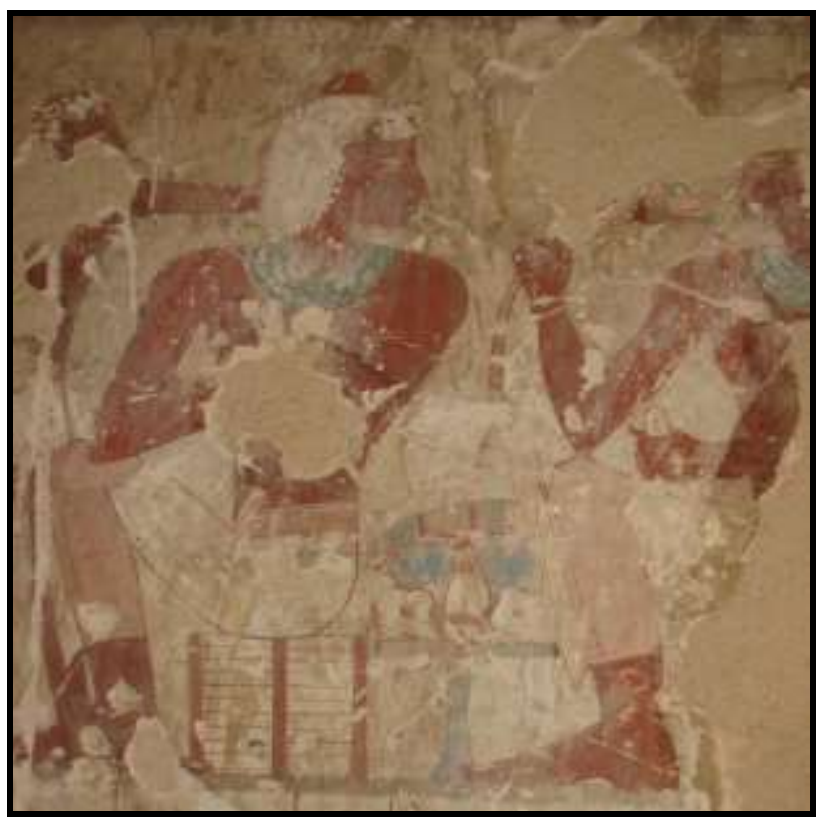

(Pl. 2)

\section{(3): La Tombe de Djéserkarâseneb $\operatorname{snbTT} 38^{31}$ :}

${ }^{29}$ Ces cônes sont constitues d'un bloc de suif ou de pommade auxquels on a mélangé de extraits de plantes odorantes. Sous l'effet de la chaleur, devait fondre et se répand dans les cheveux et sur la peau et leur donne une odeur parfumée, mais quelque fois ils faisaient des traces sur la partie supérieur des vêtements des invités, et contraire à tout ce qu'on sait de la délicatesse et de la propreté méticuleuses des anciens Egyptiens. cf. Manniche L., An Ancient Egyptian Herbal, Londres, 1989, p. 51; Germer R., «Salbkegel», LÄ V, 1984, cols. 366-367; Hepper F. N., Pharaon's flowers, Londres, 1990, p. 19; Keimer L., « Notes prises chez les Bisarins et les Nubiens d'Assouan », BIE 34, 1953, p. 367.

30 La forme de celui-ci variéra beaucoup au cours de la VIII ${ }^{\mathrm{e}}$ dynastie ; au debut de cette époque il est tres plat «au point que leur forme évoque celle d' une ampoule sur la peau» Cherpion N., «quelques jalons pour une histoire de la peinture thébaine »,BSFE 110,1987,p. 31.

31 Sa femme est 1 WAD rnpt 
دراسات في آثار الوطن العربي بـ 1

DATE: Règne de Thoutmosis IV.

SITE: Cheikh Âbd el-Gournah..

PLAN: PM I, p. 44.

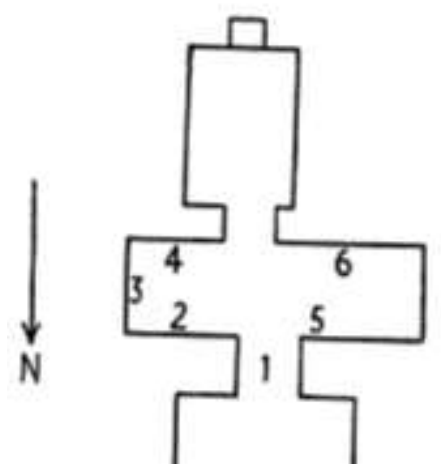

BIBLIOGRAPHIE : Scheil J. V., «Tombeau de Rat'eserkasenb » in MMAF 5/2, Paris, 1894, p. 474-6, pls. II, III ; Kuentz C., « les textes du Tombeau $\mathrm{N}^{\circ} 38$ à Thèbes (Cheikh Âbd el Gourna)» BIFAO 21, 1923, p.128-130 ; Davies N. de G., Scenes From Some Theban Tombs, $\left(N^{\circ} 38,66,162\right.$, with excerpts from 81), Oxford, 1963, p. 6, pls. V, VI; Schott photos 8551-6; MMA Photos T. 10824; PM I, p. 70 (6).

EMPLACEMENT DE LA SCÈNE: Sur le mur Sud, moitié Ouest $\left(\mathrm{n}^{\circ} 6\right)$ de la salle transversale. (Pl. 3).

LA DESCRIPTION: Dans le troisième registre de la scène du banquet on voit un des invités assis à la fin du rang, tournant sa tête pour vomir. on voit ce qu' il regette de sa bouche avec abondance. Il approche de sa main gauche un pot derrière le siège, peut-être le pot est déposé specialement pour ce but. L'artiste a bien mis en valeur les ligne de vomissement dériges vers ce pot $_{\text {s }}$ ce qui indique qu'il a vomit dans le pot, tandis que l'autre main est endommagée . l'autre invité qui est assis devant lui, est aussi tourné pour aider son

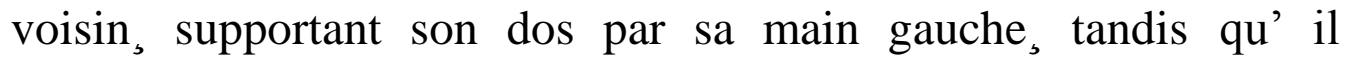
s'apprête pour soutenir sa tête par l'autre main. 


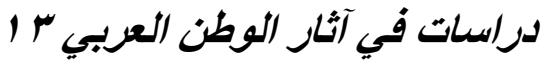

Celui qui vomis et son collège sont assis sur des tabourets ${ }^{32}$ posés ensuite sur une natte. Chacun d'eux porte un pagne court, ouvert devant, noue au milieu par une ceinture, tandis que, la partie superieur du corps est nue. Sauf un collier ousekh autour de son cou, perruque courte qui laisse dégager les oreilles.

Il est à noter que tous les invités sont peints en posant des cônes d'onguent sur leurs têtes ${ }^{33}$, sauf la personne vomissant, peut être l'artiste ne s'interresse pas à montrer ça, probablement, l'oudeur du vomissement a predominé,celui du parfume.

La scène est entourée d'une frise de khekerou où s'alternent les couleurs:rouge,vert,jaune et bleu séparées par deux traits noir et blanc.
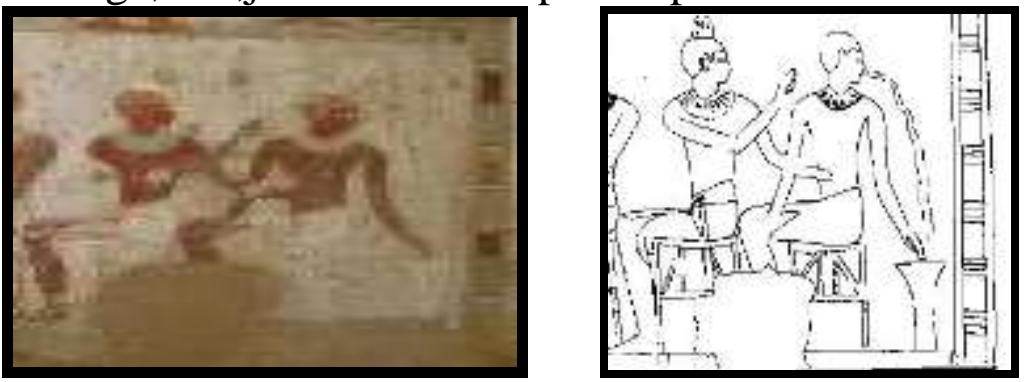

(pl. 3).

${ }^{32}$ Ces sont des tabourets simples à quatre pieds rattachés entre eux par des barres horizontales positionnées soit à mi distance des pieds soit plus bas, soit à proximité du sol ; et au centre se trouvent une ou deux baguettes de renforcement qui relient le siège à la barre horizontale, de ces baguettes verticales s'écartent en $\mathrm{V}$ renversé deux autres baguettes allant jusqu'au croisement du pied et de la barre horizontale. Ce type de tabouret ressemble aux sellettes. ce forme de tabouret est représenté dans les scènes du banquet dans plusieurs tombes. Voire: Davies N.de G., The Tomb of Nakht at Thebes, New York, 1917, pl. XV; Davies N. de G., The Tombs of Two Officials of Thoutmosis the Fourth (nos 75 and 90), Londres, 1923 pl. V; Davies N. de G., The Tomb of Two Sculptors at Thebes, New York, 1925, pl. VII; Manniche L., The Wall Decoration of Three Theban Tombs (TT77, 175 and 249), Copenhagen, 1988, fig. 3, p. 15; Guksch H., Die Gräber des Nacht-Min und des Men-cheper-Ra-Seneb: Theben Nr. 87 und 79, AVDAIK 34, 1995, 37, 38; Cherpion N., MIFAO 114, pl. 38,39. pour savoir plus cf. Hanafy D., Les sièges et les tabourets dans l' Égypte ancienne Jusqu'à la fin du Nouvel Empire, Thèse du doctorat, inpublié, (en arabe), Le Caire, 2005, p. 31f; Baker H., Furniture in the Ancient World, New York, 190; Wanscher O., The Art of Furniture, Londres, 1966.

${ }^{33}$ La forme de cône d'onguent est très nette d'un oeuf. En effect le cône de parfum s'allonge, pour devenir de plus en plus pointu. C'est dans les tombes où l'on trouve le nom de Touthmosis IV que le cône prend la forme d'un œuf, cf. Cherpion N., BSFE 110, p. 39. 


\section{(4): Un fragment de tombe Thébaine ${ }^{34}$ :}

DATE: de la XVIII ${ }^{\mathrm{e}}$ dynastie $^{35}$.

SITE: Inconnu.

DIMENSION : H. 30 cm, L. 32. cm..

CONSERVATION: Musées royaux d'art et d'histoire, Bruxelles, inv. E.2877.

BIBLIOGRAPHIE: Inpublié ${ }^{36}$.

LA DESCRIPTION : C'est une part de la scène du banquet, les traits les plus marquants de cette scène sont l'étonnante fraicheur des coloris qui donne l'impréssion d'avoir été peinte heir. Comme presque chaque fois que la peinture est appliquée sur la mouna (enduit de terre et de paille) couverte par le stuc.

34 Je remercie Dr. Luc Delvaux, conservateur des musées royaux d'art et d'histoire de Bruxelles pour sa bonne coopération de m'avoir fourni les informations suivantes : Ce fragement a été apporté du Caire en 1909 par Jean Capart, ancien conservateur des musées royaux d'art et d'histoire de Bruxelles. Il l'a acheta d'un marchand d'art bien connu de l'époque, Nahman. Certaines tombes Thébaines montrent des scènes semblables, mais il n'a malheureusement pas été possible de savoir à quelle tombe appartient ce fragment, mais il est daté à la $18^{\mathrm{e}}$ dynastie.

35 Ce fragment ne présent aucun repère de chronologie absolue, mais il y a quelque nombre $\mathrm{d}$ indices montrent que la tombe n' est pas postérieure à Amenhotep II. Ce sont:

La forme du cône d'onguent sur la tête de l'homme qui vomit et la damme qui est assise devant lui a côte de son marie (endommagé), comparable a une boule aplatie ou a une loupe sur la peau;

Le robe étroite, gainante, maintenue sur les épaule par une seule bretelle qui ressemble les robes des dames de Moyen Empire

La fleur de lotus qui orne le front des dames: celle-ci est représentée de façon si raide et si massive qu'elle évoque davantage une lampe de mineur

Le silhouettes féminines qui est dépourvue de forme, gracile, voire d'une étonnante maigreur L'opposition très forte entre la carnation des hommes et celle des femmes: les messieurs ont d'un beau brun cuivré.

Tous ces aspects indiquent que ce fragment peut dater de la première moitié de la XVIIIe dynastie, de façon plus précise du règne de Thoutmosis III, mais l'apparence de la perruque enveloppante -qui succède la perruque tripartite et qui est apparue pour la première fois sous le règne du Amenhotep II- nous donne le tendance a dater ce fragement de la fin du règne du Thoutmosis III.

${ }^{36}$ Capart J., in: Bull. Des Mus. 2 sér. III, 1910, p. 41, fig. 7; Wreszinski W., Atlas zur altagyptischen Kulturgeschichte, I, Leipzig, 1923, Tf. 392; PM I, 815; L. Manniche, Lost Tombs. A study of certain Eighteenth Dynasty Monuments in the Theban Necropolis, Londres \& New York, 1988, p. 188; Reeves C., Egyptian Medicine, Londres, 2001, p..14, fig. 3. 


\section{دراسات في آثار الوطن العربي بـ 1}

On voit un des invités assis sur un tabouret de forme cubique de hauteur moyenne ${ }^{37}$; tournant sa tête pour vomir. on voit ce qu' il regette de sa bouche avec abondance, probablement il n'y pas du pot à côté de lui pour vomir dedans. l'autre invité qui est assis près de lui, est tourné vite -dans un mouvement étrange qu'on voit pour la première fois dans les tombes Thébaines pour aider son voisin ${ }^{38}$, il est en train de poser la main droite sur la tête et en posant sa main gauche sur son bras gauche pour le supporter pendant le vomissement.

Ils portent deux pagnes superposés, un court à devanteau triangulaire et un long transparent ${ }^{39}$; autour de la taille, il y a une ceinture festonnée. tandis que, la partie superieur du corps est nue. Sauf un collier ousekh autour de son cou, perruque courte qui laisse dégager les oreilles. Sur laquelle est posé un cône d'onguent.

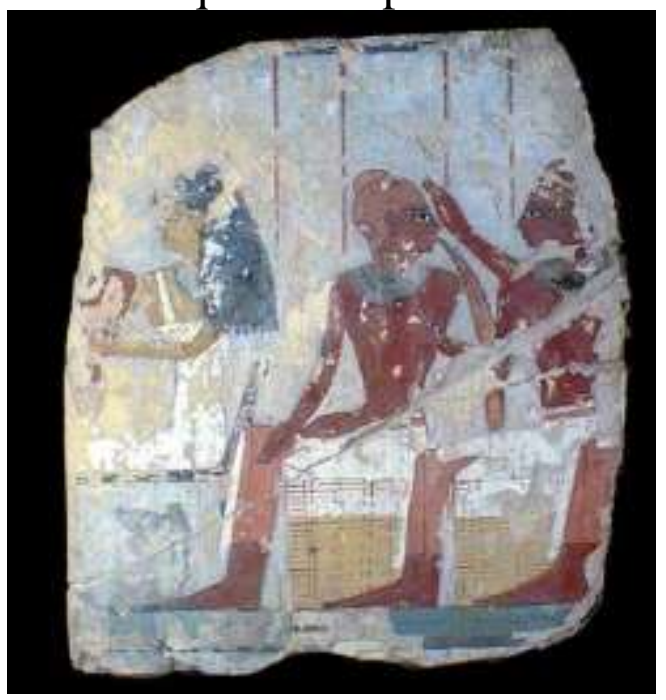

(pl. 4)

${ }^{37}$ Il ressemble a celui qu' on a deja vue dans la tombe TT84, cf., supra. P1. 2

${ }^{38} \mathrm{Il}$ parait pour le voyant le dos et pas le ventre, l'arme droite semble qu'elle sortit de l'épaule gauche. Habituellement, si l'artiste a voulut montrer l'operation du tournement, il le montre avec la position naturelle des deux bras et pas comme ce geste illogique.

${ }^{39}$ l'artiste utilise du rose pâle par opposition aux zones d'un blanc opaque qui indiquent une double epaisseur de tissu. 
دراسات في آثار الوطن العربي 1 آ

(B): Les scènes du vomissement dans les tombes des particuliers de la XIX ${ }^{\mathrm{e}}$ dynastie :

(1): La Tombe de Néferhotep $\stackrel{t}{\square}$ nfr-HtpTT $49^{40}$ :

DATE: Règne d'Aÿ.

SITE: El- Khôkha.

BIBLIOGRAPHIE: Davies N. de G., The Tomb of Nefer-Hotep at Thebes, New York, 1933, p. 26-7, pl. V, pl. XVIII. PM I, p. 92 (6). PLAN: PM I, p. 90.

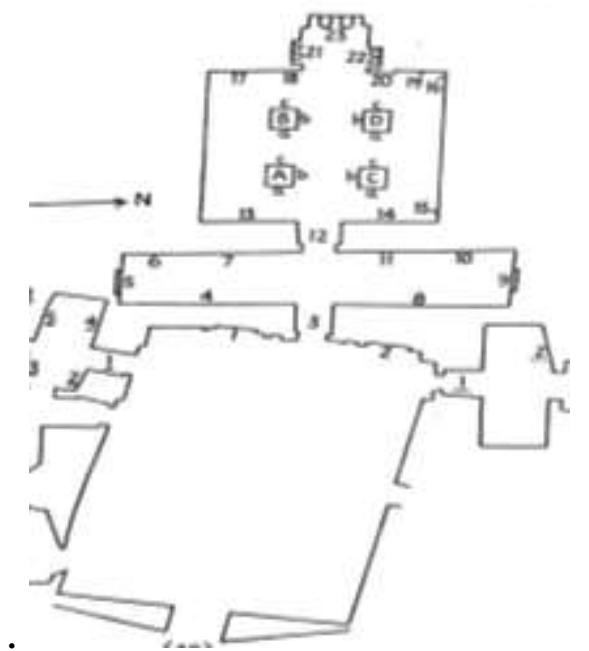

EMPLACEMENT DE LA SCÈNE: Sur la paroi Ouest, partie Sud $\left(\mathrm{n}^{\circ} 6\right)$ de la salle transversale. (Pl. 5).

LA DESCRIPTION: Sur le registre supérieur de la scène du banquet, on voit une des invités assise sur un petit banc au bout du rang. Elle tourne sa tête en arrière vomissant sur terre parce que il n' y avait pas de pot à coté d'elle. On croirait qu'elle n'a pas eu le temps d'attendre la servante qui accourait pieds nus posant les sandales en dessous de son bras droite, pour les enfiler plus tard, une preuve qu'elle est venue vite pour aider sa maîtresse. Pendant

40 Son titre principle est

Gardiner A. \&A. Weigall A., op. Cit., p. 20; Taylor J. A., op. Cit., p. 209 (3036). Ses parents

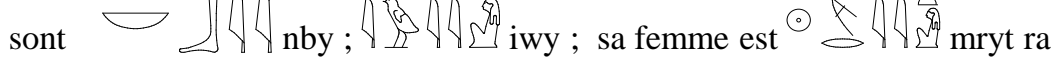




\section{دراسات في آثار الوطن العربي بـ 1}

qu'elle tient avec la même main le pot, elle se penche pour l'approcher à la bouche de la dame pour en vomir. La servante en question met sa main gauche sur la tête de la dame pour la soutenir pendant le vomissement.

l'invitée assise à côté d' elle s'intéressait de ce qu' il arrive . Elle s'est tournée pour aider sa voisine en posant la main droite sur son épaule alors que l'autre main est posée mollement sur le genou en tenant une branche d'une plante au lieu de la fleur de lotus habituelle. Ce que se convient avec l'ambiance du banquet, qui est organisé en plein aîr, dans une salle précédée d'un jardin du palais, où on voit des hommes cueillir des fruits.

Les femmes sont vêtues de longues robes transparentes, plissées, sans manches, avec un châle sur le bras droit ${ }^{41}$ et coiffées des perruques longues tripartites avec un serre tête noué à l'arrière avec une fleur de lotus et un cône d'onguent traversé par une fleur de lotus. Tandis que la servante porte la même robe transparente qui découvre ses jambes, alors qu' elle est débout, cette robe a manche courte et large et portant une perruque longue.

${ }^{41}$ Cette robe est née avec l' époque amarnienne mais, elle est beaucoup plus ample, plus vaste qu'auparavant. Ceci correspond peut-être à une réalité, mais peut-être aussi les artistes du temps en ont-ils exagéré simplement l' ampleur car l'époque ramesside aime les ieux graphiques. Cherpion N., BSFE 110, 1987, p. 27-47. et par example Lhote A., Les chefsd'œuvre de la peinture égyptienne, Paris, 1954. pls. 131, 132,134,136; Mekhitarian A., «La destruction systématique des tombes thébaines » in : Mélanges offerts à Jean Vercoutter, Paris, 1985, p. 239-241;.Mekhitarian A., «La tombe de Nebamon et Ipouki (T T181) » in Tefnin R. (ed.) La peinture égyptienne ancienne. Un monde de signes préserver, Actes du colloque international de Bruxelles, Avril 1994, Bruxelles, 1997, p. 21-27; Mekhitarian A., «Un peintre thébain de la XVIIIe dynastie », MDAIK 15, 1957, p. 186-192; Mekhitarian A., «Un peintre thébain de la XVIIIe dynastie », MDAIK 15, 1957, p. 186-192; Davies N. de G., The Tomb of Two Sculptors at Thebes, New York, 1925, pls. V-VII, , pl. XXVII-XXIX; Scheil V., « Le Tombeau des graveurs » MMAF 5/2, p. 561-4, pls. IV-V . 
دراسات في آثار الوطن العربي بـ 1

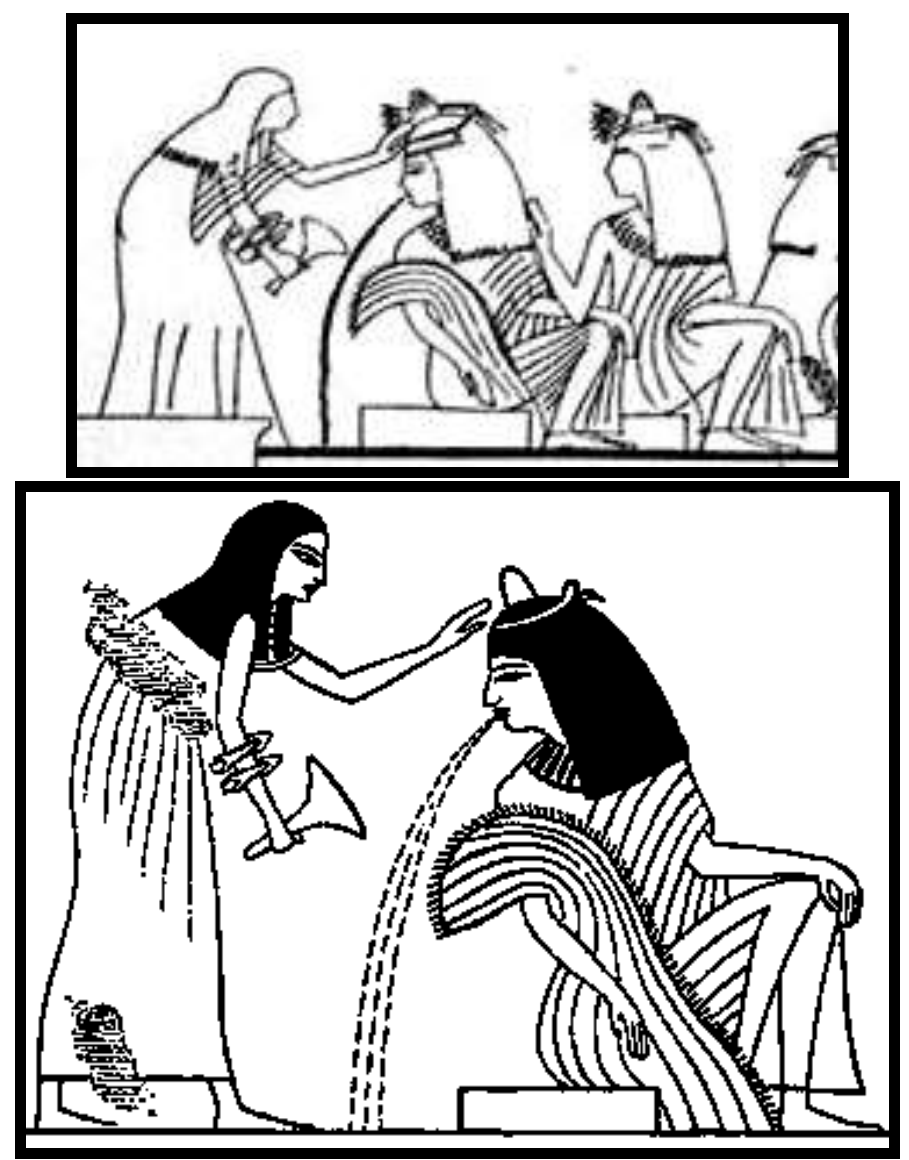

(pl. 5).

\section{(3): Les résultats :}

Au terme de cette recherche, il convient de tirer un certain nombre de conclusions :

(1) : Les scènes du vomissement trouvaient toujours sur les parois de la salle transversale de la tombe parce que l'artiste égyptien (ancien) procède selon une règle fondamentale, très répandue et présidant à la répartition des scènes de la vie quotidienne.

(2) : L'étude fait apparaître que les scènes du vomissement sont toujours accompagnées de scènes du banquet et sont jamais trouvées isolées.

(3) : A partir des scènes décrites, nous avons observé que, ces repas étaient organisés uniquement par les notables et l'élite de la société, 
et au cours desquels on consommait et dégustait une grande variété de mets et de boissons. On s'attendait à voir les personnages en train de manger puisque les victuailles étaient posées devant eux ; or ce que l'on note à travers ces scènes, représente uniquement des personnages en train de se servir à boire ou bien en train de boire. Cela signifie que les vomissements dans ces scènes peuvent être causés par consommation d'alcool.

(4) : Parfois, on provient le vomissement, l'artiste représenta les pots mis à côté des hôtes pour y vomir, enconservant le nettoyage de la place. Mais quelque fois, le vomissement se passe d'une façon accidentelle par terre. 